\title{
2-arachidonyl glycerol modulates astrocytic glutamine synthetase via p38 and ERK1/2 pathways
}

\author{
Shenghong Wang ${ }^{1+}$, Hua Zhang ${ }^{1 \dagger}$, Bin Geng ${ }^{1,2}$, Qiqi Xie ${ }^{1}$, Wenzhou $\mathrm{Li}^{1}$, Yajun Deng ${ }^{1}$, Weidong Shi ${ }^{1}$, Yunyan Pan ${ }^{3}$,
} Xuewen Kang ${ }^{1,2}$ and Jing Wang ${ }^{1 *}$

\begin{abstract}
Background: The glutamine synthetase (GS), an astrocyte-specific enzyme, is involved in lipopolysaccharide (LPS)induced inflammation which activates the mitogen-activated protein kinase (MAPK) signaling. Endocannabinoid 2arachidonyl glycerol (2-AG) has been described to serve as an endogenous mediator of analgesia and neuroprotection. However, whether 2-AG can directly influence astrocytic GS and MAPK expressions remains unknown.

Methods: In the present study, the effects of 2-AG on astrocytic GS expression, p38 and ERK1/2 expression, cell viability, and apoptosis following LPS exposure were investigated.

Results: The results revealed that LPS exposure increased GS expression with p38 activation in the early phase and decreased GS expression with activation of ERK1/2, decrease of cell viability, and increase of apoptosis in the late phase. Inhibition of p38 reversed GS increase in the early phase while inhibition of ERK1/2 reversed GS decrease in the late phase induced by LPS exposure. 2-AG protected astrocytes from increase of apoptosis and decrease of cell viability induced by the late phase of LPS exposure. In the early phase of LPS exposure, 2-AG could suppress the increase of GS expression and activation of p38 signaling. In the late phase of LPS exposure, 2-AG could reverse the decrease of GS expression and activation of ERK1/2 induced by LPS.

Conclusion: These findings suggest that 2-AG could maintain the GS expression in astrocytes to a relatively stable level through modulating MAPK signaling and protect astrocytes from LPS exposure.
\end{abstract}

Keywords: 2-AG, Astrocyte, Glutamine synthetase, MAPK, p38, ERK

\section{Background}

Astrocytes are the main glial cells in the central nerve system (CNS) where they play a central role in neurotrophic support, regulation of the concentration of extracellular ions, antioxidant defense, and neurotransmitter metabolism [1]. In astrocytes, glutamine synthetase (GS), an ATP-dependent enzyme, could modulate the extracellular level of glutamate, an essential but neurotoxic excitatory neurotransmitter, by converting glutamate to nontoxic amino acid glutamine [2]. Changes in GS expression have been identified in a number of neurological

\footnotetext{
* Correspondence: wang_jing@|zu.edu.cn

†'Shenghong Wang and Hua Zhang contributed equally to this work.

${ }^{1}$ Key Laboratory of Orthopaedics Disease of Gansu Province, Lanzhou

University Second Hospital, No.82 Cuiyingmen Street, Lanzhou, Gansu

730030, People's Republic of China

Full list of author information is available at the end of the article
}

disorders, including traumatic brain injury, neurodegenerative diseases, and various models of nociceptive pain, while modulating GS expression could diminish relative clinical features [3-8]. Endocannabinoids are endogenous lipid signaling mediators capable of modulating synaptic function and producing neuroprotection and anti-inflammation [9, 10]. 2-arachidonoyl glycerol (2-AG), the most abundant endocannabinoid, has been illustrated to play a significant role in protecting neurocytes from inflammatory injuries, such as stimuli of interleukine-1 beta (IL-1 $\beta$ ), lipopolysaccharide (LPS), and $\beta$-amyloid in models of neurodegenerative diseases [11, 12]. Besides, 2-AG has been shown to have the ability of controlling neuropathic pain and mechanical hyperalgesia in several preclinical models of chronic pain [13-16]. Although the role of 2-AG has been extensively investigated, it is not clear

(c) The Author(s). 2018 Open Access This article is distributed under the terms of the Creative Commons Attribution 4.0 International License (http://creativecommons.org/licenses/by/4.0/), which permits unrestricted use, distribution, and reproduction in any medium, provided you give appropriate credit to the original author(s) and the source, provide a link to the Creative Commons license, and indicate if changes were made. The Creative Commons Public Domain Dedication waiver (http://creativecommons.org/publicdomain/zero/1.0/) applies to the data made available in this article, unless otherwise stated. 
whether 2-AG could directly modulate the GS expression in astrocytes, and the exact molecular mechanism remains unknown.

The mitogen-activated protein kinase (MAPK) cascades are a family of serine/threonine kinases that can mediate a wide variety of extracellular stimuli into the cytoplasm and nuclei and regulate cellular gene expression and protein synthesis [17]. Previous studies have indicated that MAPK members, extracellular signal-regulated protein kinase 1/2 (ERK1/2) and p38 pathways, in astrocytes may be involved in the process of various neurological disorders [18]. Accumulating evidence showed that activation (phosphorylation) of ERK1/2 and p38 in spinal astrocytes under different persistent pain conditions results in the generation and maintenance of pain hypersensitivity via distinct molecular and cellular mechanisms [19]. In addition, activation of ERK1/2 and p38 in cortical astrocytes has been identified in several neurodegenerative diseases, while blockade of ERK1/2 and p38 pathways has been shown to alleviate inflammation and clinical features in different animal models [20-22].

It has been verified that 2 -AG could modulate synaptic function, produce neuroprotection, and stimulate MAPK family by bonding to and activating two receptors, $C_{1} R$ and $\mathrm{CB}_{2} \mathrm{R}$, which are two G-protein-coupled receptors and have been grossly identified in astrocytes [23]. Previous studies have shown that activation of $\mathrm{CB}_{1} \mathrm{R}$ or $\mathrm{CB}_{2} \mathrm{R}$ results in anti-inflammation, prevention of neurodegeneration, and inhibition of nociceptive signaling pathways [24-26], as well as stimulation of the MAPK cascade [27-29]. Interestingly, activation of $\mathrm{CB}_{1} \mathrm{R}$ or $\mathrm{CB}_{2} \mathrm{R}$ also could inhibit stress-induced activation of the MAPK cascade. Based on these findings, it is proposed that 2-AG may exert neuroprotection and analgesia via activating $\mathrm{CB}_{1} \mathrm{R}$ or $\mathrm{CB}_{2} \mathrm{R}$ and regulating activation of the MAPK cascade.

\section{Methods}

\section{Primary astrocyte cultures}

Primary astrocytes from the cerebral cortex of neonatal Sprague-Dawley rats (postnatal 1 3 days) were cultured as described preciously [30]. The neonatal SpragueDawley rats were provided by the Experimental Animal Center of Gansu University of Chinese Medicine, China. All efforts were performed to minimize the number of neonatal rats used and their suffering. The procedures were approved by the Animal Care and the Ethic Committee of Animal Usage of Lanzhou University Second Hospital. Briefly, the newborn rats were decapitated and the cerebral hemispheres were aseptically removed into HBSS (Hank's Balanced Salt Solution). After removal of the meninges, the cerebral cortices were cut into small pieces, digested with $0.25 \%$ Trypsin-EDTA (Gibco Life Technology, CA, USA), mechanically dissociated by gentle pipetting with Pasteur pipette, and then centrifuged at $400 \mathrm{~g}$ for $5 \mathrm{~min}$. The cells were resuspended in complete culture medium containing 90\% DMEM/F12 (Gibco Life Technology, CA, USA) and 10\% FBS (PAN-Biotech, Germany), and plated at a density of $3 \sim 5 \times 10^{5}$ cells $/ \mathrm{cm}^{2}$ in $25-\mathrm{cm}^{2}$ flasks. Cells in flasks were cultured at $37{ }^{\circ} \mathrm{C}$ in $\mathrm{CO}_{2}$ incubator for $5 \sim 7$ days to reach the first confluence. To obtain quite pure astrocytes (more than $95 \%)$, the confluent cultures in flasks were shaken at $200 \mathrm{rpm}$ overnight to diminish microglia contamination. Afterward, the astrocytes were evenly passaged into 35-mm dishes for western blot analysis, on coverslips pre-coated with poly-L-lysine for immunocytochemistry analysis, and into 96-well plates for methyl thiazolyl tetrazolium (MTT) analysis after different treatments. Astrocytes were cultured with serum-free medium for $6 \mathrm{~h}$ before different treatments.

\section{Drugs treatment}

All drugs were dissolved and/or diluted with serum-free DMEM/F12 into final concentration. To study the effect of 2-AG, astrocytes were incubated with $0.01 \mu \mathrm{M} 2$ 2-AG for $2 \mathrm{~h}$ and then stimulated with $1 \mu \mathrm{g} / \mathrm{ml}$ LPS. To study the roles of ERK1/2 and p38 in LPS-induced inflammation, astrocytes were pretreated with PD98059 or SB203580 for $1 \mathrm{~h}$ before LPS exposure. To study the roles of $C_{1} R$ and $C_{2} R$ on effects of 2-AG, astrocytes were pretreated with $1 \mu \mathrm{M}$ AM281 or AM630 for $1 \mathrm{~h}$ before the treatment of 2-AG and LPS.

\section{Hoechst 33342 staining}

After various treatments, astrocytes were stained with Hoechst 33342 kit (St. Louis, MO, USA) and counted blindly as described previously [31]. Briefly, astrocytes on coverslips were rinsed with PBS and fixed with $4 \%$ paraformaldehyde for $30 \mathrm{~min}$. After being rinsed three times with PBS, astrocytes were incubated with $0.4 \%$ Triton X-100 for $20 \mathrm{~min}$, and then stained with Hoechst 33342 for $10 \mathrm{~min}$ in the dark. After washing cells with PBS, the nuclear morphological changes of apoptosis were observed using a fluorescence microscope (Olympus, Japan). Astrocytes with bright staining, highly condensed and fragmented nuclei were defined as apoptotic cells. The number of apoptotic cells and total cells were counted, and then the cell apoptosis rate was calculated by the following equation:

Cell apoptosis rate $(\%)=\left(N_{\text {apoptotic cells }} / N_{\text {total cells }}\right) \times 100$

\section{Methyl thiazolyl tetrazolium (MTT) assay}

Cell viability was detected using 3-(4,5-dimethythiazo1-2-yl)-2,5-diphenyl-tetrazolium (MTT) assay as described previously [31]. In brief, astrocytes were cultured 
in 96-well plates at a density of $3 \times 10^{4}$ cells/well. After different treatments, astrocytes were incubated with $1 \mathrm{mg} / \mathrm{ml}$ substrate MTT at $37^{\circ} \mathrm{C}$ for $4 \mathrm{~h}$. Then the culture medium was replaced by $100 \mu \mathrm{l}$ dimethyl sulfoxide (DMSO) to dissolve the formazan crystals. The amount of formazan was measured at $570 \mathrm{~nm}$ using a Universal Microplate Reader (Elx 800; Bio-TEK instruments, Winooski, VT, USA). The cell viability was expressed as a percentage of viable cells in treated groups versus a control group using the following formula:

$$
\begin{aligned}
\text { Cell viability }(\%)= & \left(\text { Opticaldensity }_{\text {treatment }}\right. \\
& \left./ \text { Opticaldensity } \text { control }_{\text {Opl }}\right) \times 100
\end{aligned}
$$

\section{Protein isolation and western blotting}

Western blotting was performed according to a previous report [32]. Briefly, astrocytes in 35-mm dishes were lysed with $100 \mu \mathrm{l}$ radioimmunoprecipitation assay (RIPA) lysis buffer containing $1 \%$ phenylmethanesulfonyl fluoride (PMSF) after different treatments. The lysates were centrifuged at 12,000 rpm for $10 \mathrm{~min}$ to clear cell debris and further diluted with $30 \mu \mathrm{l}$ sample buffer. Total protein in lysates was loaded onto 10\% SDS-polyacrylamide gels at 5-20 $\mu \mathrm{g}$ per lane (as measured by BCA), then separated by electrophoresis and transferred to PVDF membranes. Following the blockade of nonspecific binding sites with $5 \%$ non-fat milk in Tris-buffered saline with Tween-20 (TBST) for $2 \mathrm{~h}$ at room temperature (RT), the membranes were incubated overnight at $4{ }^{\circ} \mathrm{C}$ with primary antibodies according to the manufacturer's instruction (anti-MAPK antibody, 1:1000, Cell Signaling Technology, MA, USA; anti-GS antibody, 1:10,000, St. Louis, MO, USA) and then washed extensively with TBST three times at 10-min intervals and incubated with appropriate second antibodies (1:10,000; Danvers, MA, USA) at RT for $2 \mathrm{~h}$. The membranes were washed three times with TBST at 10-min intervals, and immunolabeled protein bands on membranes were detected using an enhanced chemiluminescence kit.

\section{Immunocytochemistry}

After different treatments, the astrocytes on coverslips were fixed with $4 \%$ paraformaldehyde for $30 \mathrm{~min}$ and washed with PBS. The fixed cells were permeabilized with $0.4 \%$ TritonX-100 for $20 \mathrm{~min}$, washed again with PBS, blocked with $3 \%$ normal goat serum for $30 \mathrm{~min}$, and then incubated with different primary antibodies (GS, 1:5000; ERK1/2, 1:500; p38, 1:500) overnight at $4{ }^{\circ} \mathrm{C}$, respectively. After $24 \mathrm{~h}$, the coverslips were washed and incubated with appropriate second antibodies (Invitrogen, UK) conjugated with Alexa Fluor 488 (green staining) or 594 (red staining) for $2 \mathrm{~h}$ at RT. All cells were stained with
DAPI for nuclei observation, and cells were visualized by an immunofluorescence microscope (Olympus, Japan).

\section{Statistics analysis}

All experiments were carried out in triplicate and repeated at least three times, and all measurements were performed by blinded evaluators. The data were expressed as mean \pm standard deviation (SD), and STATA software (version 14.2, Stata Corp, College Station, TX, USA) was used for statistical analysis. One-way ANOVA followed by Neuman Keuls test was mainly performed, and two-way ANOVA followed by Dennett test was performed in Fig. 4; $p<0.05$ was set as the level of significant difference.

\section{Results}

LPS activated rat primary astrocyte dosage-dependently and GS time-dependently

Astrocyte activation is a common outcome of neurological disorders. In the present study, LPS was used to activate astrocytes. Cells were incubated in DMEM/F12 containing LPS at various concentrations $(0,0.01,0.1$, and $1 \mu \mathrm{g} / \mathrm{ml}$ ) for $6 \mathrm{~h}$, and GFAP expression was analyzed using western blotting to assess the activation of astrocytes (Fig. 1a). The data showed that compared with control $(1.00 \pm 0.12$ for $0 \mu \mathrm{g} / \mathrm{ml}$ of LPS $)$, the expression of GFAP in astrocytes with LPS treatment was significantly increased in a dose-dependent manner $(1.68 \pm$ 0.13 for $0.01 \mu \mathrm{g} / \mathrm{ml}, p<0.05 ; 2.52 \pm 0.20$ for $0.1 \mu \mathrm{g} / \mathrm{ml}, p$ $<0.01 ; 3.71 \pm 0.25$ for $1 \mu \mathrm{g} / \mathrm{ml}, p<0.001)$.

In order to investigate the effects of LPS exposure on GS expression, astrocytes were exposed to $1 \mu \mathrm{g} / \mathrm{ml}$ LPS for different times and the level of GS expression was assessed using western blotting (Fig. 1b). The results indicated that LPS exposure induced time-dependent biphasic changes of GS expression in astrocytes; i.e., compared with control $(1.00 \pm 0.07$ for $0 \mathrm{~min})$, GS expression began to increase at $30 \mathrm{~min}(2.26 \pm 0.06, p<$ $0.001)$, peaked at $1 \mathrm{~h}(3.15 \pm 0.10, p<0.001)$, declined to control level at $3 \mathrm{~h}(1.12 \pm 0.10, p>0.05)$, and then decreased at $6 \mathrm{~h}(0.32 \pm 0.05, p<0.01), 12 \mathrm{~h}(0.18 \pm 0.02$, $p<0.001)$, and $24 \mathrm{~h}(0.06 \pm 0.03, p<0.001)$.

\section{Late phase of LPS exposure decreased astrocyte viability time-dependently}

To investigate the effect of LPS on astrocyte viability, astrocytes were exposed with $1 \mu \mathrm{g} / \mathrm{ml}$ LPS for different times and astrocyte viability was assessed with MTT assay (Fig. 1c). The data indicated that LPS exposure decreased the astrocyte viability at the late phase, i.e., astrocyte viability decreased at $6 \mathrm{~h}(0.60 \pm 0.01, p<0.001), 12 \mathrm{~h}(0.45 \pm$ $0.01, p<0.001)$, and $24 \mathrm{~h}(0.43 \pm 0.01, p<0.001)$ when compared with control $(1.00 \pm 0.01$ for $0 \mathrm{~min})$, while the cell viability of astrocytes at the early phase $(30 \mathrm{~min}, 1 \mathrm{~h}, 2 \mathrm{~h}$, and $3 \mathrm{~h}$ ) was not significantly changed ( $p>0.05$, Fig. 1c). 


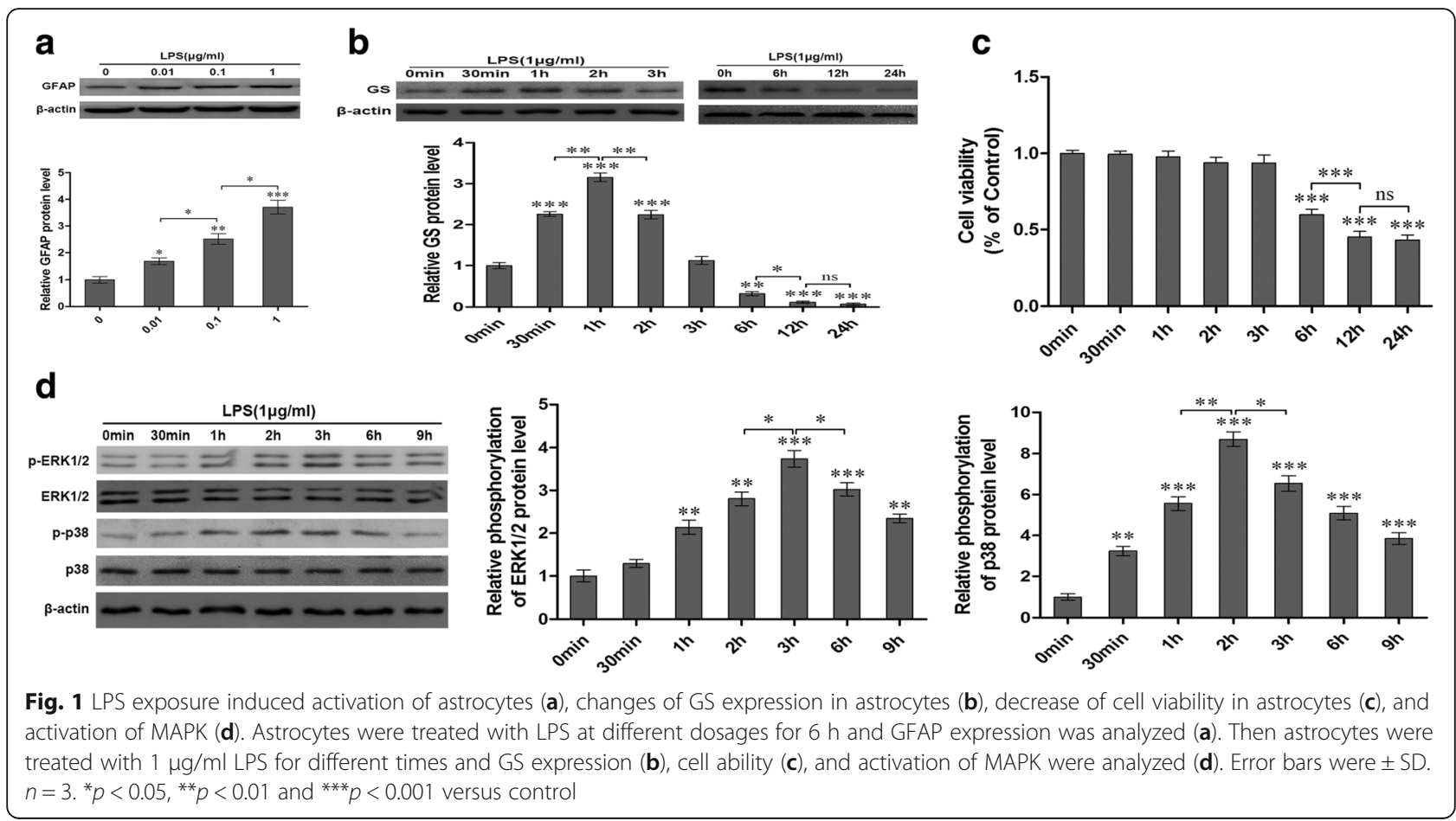

\section{LPS activated the phosphorylation of p38 and ERK $1 / 2$ in astrocytes}

To investigate the effects of LPS on MAPK cascade in astrocytes, the expression of p-ERK1/2, ERK1/2, p-p38, and p38 in primary astrocytes was investigated using western blotting after treatment with $1 \mu \mathrm{g} / \mathrm{ml}$ LPS for $0 \mathrm{~min}, 30 \mathrm{~min}, 1 \mathrm{~h}, 2 \mathrm{~h}, 3 \mathrm{~h}, 6 \mathrm{~h}$, and $9 \mathrm{~h}$ (Fig. 1d). The data showed that LPS exposure significantly increased the phosphorylation of ERK1/2 and p38 in different patterns without effect on the total ERK1/2 and p38. The expression of $\mathrm{p}-\mathrm{ERK} 1 / 2$ began to increase at $1 \mathrm{~h}(2.14 \pm$ $0.17, p<0.01)$ and gradually reached peak at $3 \mathrm{~h}(3.74 \pm$ $0.20, p<0.001)$, then declined but was still higher at $9 \mathrm{~h}$ $(2.34 \pm 0.10, p<0.01)$ than control. The expression level of p-p38 increased at $30 \mathrm{~min}(3.24 \pm 0.23, p<0.01)$ and reached to peak at $2 \mathrm{~h}(8.70 \pm 0.35, p<0.001)$, and then gradually declined but was still higher at $9 \mathrm{~h}(3.85 \pm 0.27$, $p<0.001$ ) than control.

\section{2-AG protected astrocytes from apoptosis and decrease} of cell viability induced by the late phase of LPS exposure To evaluate the potential protective roles of $2-A G$ in cultured astrocytes, the astrocytes were pretreated with $0.01 \mu \mathrm{M}$ 2-AG for $2 \mathrm{~h}$ and then exposed to $1 \mu \mathrm{g} / \mathrm{ml} \mathrm{LPS}$ for $12 \mathrm{~h}$. The Hoechst 33343 staining (Fig. 2a) and corresponding statistical chart (Fig. $2 \mathrm{~b}$ ) showed that 2-AG significantly attenuated the cell apoptosis induced by LPS exposure $(2.16 \pm 0.58$ for 2 -AG plus LPS vs. $8.75 \pm$ 0.77 for LPS alone, $p<0.001)$. Similarly, MTT assay (Fig. 2c) indicated that 2-AG significantly reversed the decrease of cell viability induced by LPS exposure $(0.84$ \pm 0.02 for 2 -AG plus LPS vs. $0.45 \pm 0.02$ for LPS alone, $p$ $<0.001)$. Bax and Bcl-xl are two members of Bcl-2 family of proteins. Bax acts as a pro-apoptotic regulator and $\mathrm{Bcl}-\mathrm{xl}$ acts as an anti-apoptotic regulator. The western blotting results (Fig. 2d) and corresponding statistical chart (Fig. 2e) showed that 2-AG significantly reversed the decrease of Bcl-xl $(0.94 \pm 0.04$ for 2 -AG plus LPS vs. $0.57 \pm 0.02$ for LPS alone, $p<0.001)$ and the increase of $\operatorname{Bax}(0.79 \pm 0.09$ for 2 -AG plus LPS vs. $1.61 \pm 0.04$ for LPS alone, $p<0.001$ ) induced by LPS exposure.

\section{Inhibition of p38 attenuated GS upregulation in} astrocytes with the early phase of LPS exposure

To validate the potential involvement of p38 signaling pathway in the regulation of GS expression in astrocytes, the astrocytes exposed to $1 \mu \mathrm{g} / \mathrm{ml}$ LPS for $2 \mathrm{~h}$ was chosen on the basis on the acquired data from Fig. 1. Pretreatment with p38 inhibitor SB203580 at the concentration of 1,5 , and $10 \mu \mathrm{M}$ for $1 \mathrm{~h}$ significantly reversed the phosphorylation level of p38 induced by LPS $(8.03 \pm 0.85, p<$ $0.01 ; 2.55 \pm 0.82, p<0.001 ; 5.88 \pm 0.57, p<0.001$, respectively) when compared with LPS alone group (16.58 \pm 0.69 , Fig. 3a), and the inhibition of $10 \mu \mathrm{M}$ LPS was weaker than $5 \mu \mathrm{M}$ LPS $(p<0.01)$. As expected, SB203580 at the concentration of 1,5 , and $10 \mu \mathrm{M}$ also suppressed the upregulation of GS induced by LPS exposure at the early phase (2 h) $(1.12 \pm 0.05, p<0.05 ; 0.51 \pm 0.04, p<0.001 ; 0.48 \pm$ $0.05, p<0.001$, respectively) when compared with LPS alone group (1.40 \pm 0.07 , Fig. 3a). 


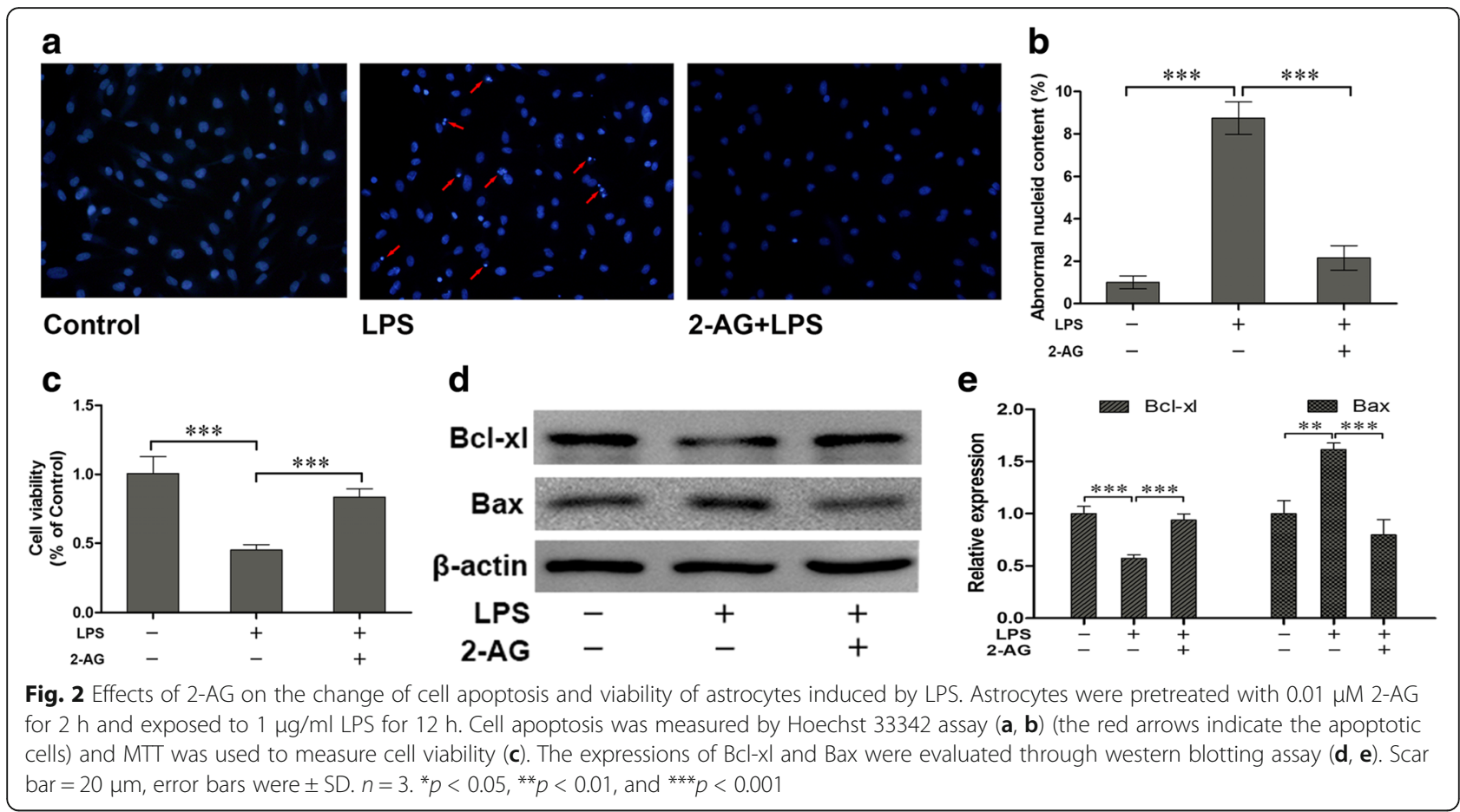

Inhibition of ERK1/2 reversed GS downregulation in astrocytes by the late phase of LPS exposure

To further investigate the effects of ERK1/2 on GS downregulation induced by LPS, LPS exposure for $12 \mathrm{~h}$ was chosen in view of data from Fig. 1 and astrocytes were pretreated with ERK1/2 inhibitor PD98059 at the concentration of 20,50 , and $100 \mu \mathrm{M}$ for $1 \mathrm{~h}$. The results indicated that PD98059 significantly inhibited the phosphorylation of ERK1/2 induced by LPS in a dose-dependent manner $(2.74 \pm 0.10$ for $20 \mu \mathrm{M}, p<0.01$; $2.03 \pm 0.08$ for $50 \mu \mathrm{M}, p<0.01 ; 1.32 \pm 0.11$ for $100 \mu \mathrm{M}$, $p<0.01$, respectively) when compared with LPS alone

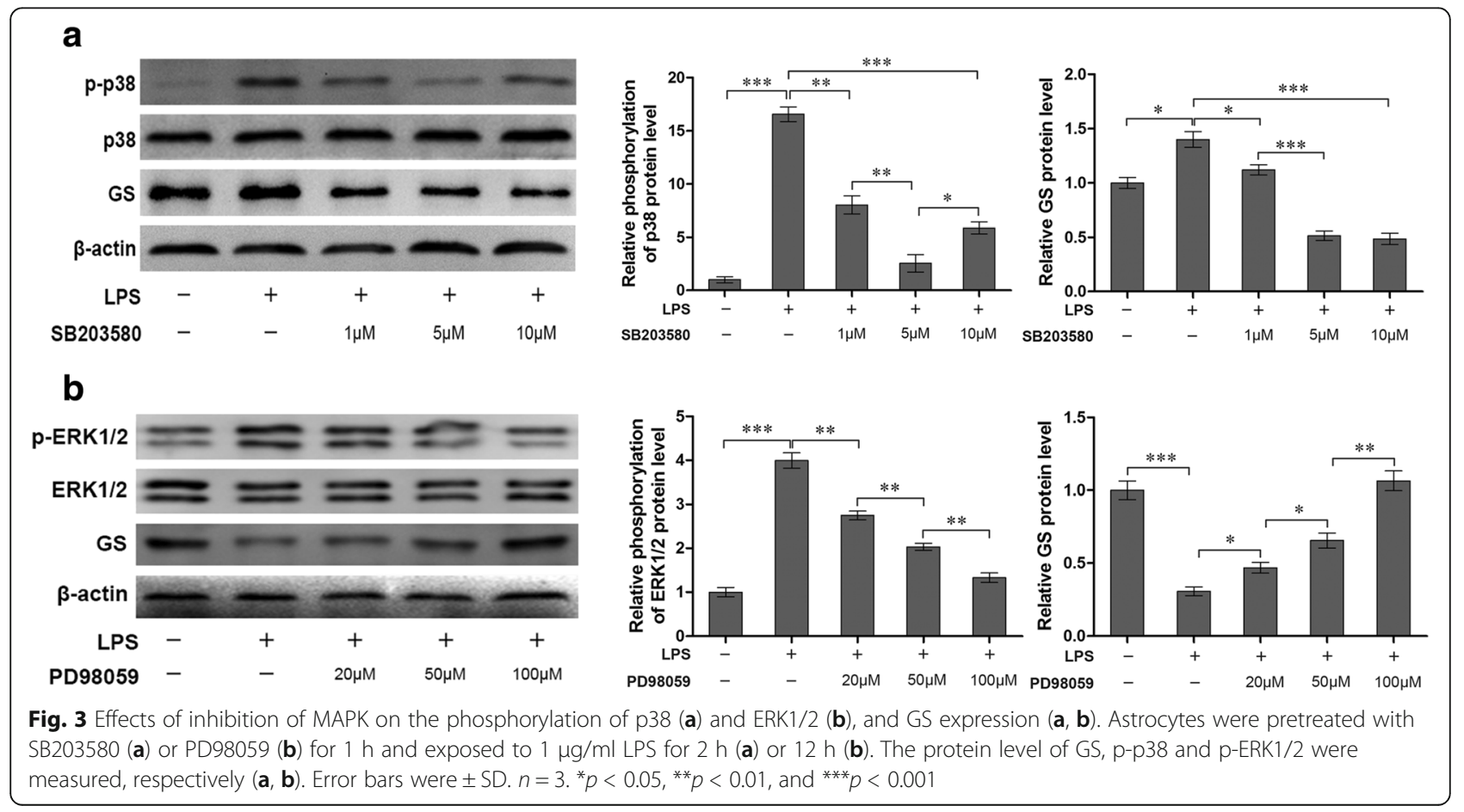


group ( $3.99 \pm 0.18$, Fig. 3b). Furthermore, PD98059 pretreatment could reverse the downregulation of GS expression induced by LPS exposure at late phage $(0.47$ \pm 0.04 for $20 \mu \mathrm{M}, p<0.05 ; 0.66 \pm 0.05$ for $50 \mu \mathrm{M}, p<$ $0.05 ; 1.06 \pm 0.07$ for $100 \mu \mathrm{M}, p<0.01$; respectively), in a dose-dependent manner, when compared to LPS alone group $(0.31 \pm 0.03$, Fig. $3 b)$.

\section{2-AG suppressed p38 activation and GS upregulation induced by the early phase of LPS exposure}

To investigate the effects of 2-AG on activation of astrocytes induced by the early phase of LPS exposure, the cells were pretreated with $0.01 \mu \mathrm{M}$ 2-AG for $2 \mathrm{~h}$ and $/$ or $1 \mu \mathrm{g} / \mathrm{ml}$ LPS for $2 \mathrm{~h}$. Compared with control, exposure of astrocytes to LPS significantly elevated the expressions of p-ERK $1 / 2$ $(1.40 \pm 0.11$ for LPS vs. $1.00 \pm 0.07$ for control, $p<0.05)$, p-p38 (13.14 \pm 1.47 for LPS vs. $1.00 \pm 0.45$ for control, $p<$ $0.001)$, and GS $(2.09 \pm 0.18$ for LPS vs. $1.00 \pm 0.08$ for control, $p<0.001)$. 2-AG could significantly reverse the LPS-induced changes of p-p38 $(5.64 \pm 1.31$ for 2 -AG plus LPS vs. $13.14 \pm 1.47$ for LPS, $p<0.01)$ and GS $(1.11 \pm 0.09$ for 2-AG plus LPS vs. $2.09 \pm 0.18$ for LPS, $p<0.001)$ when compared with LPS alone group, but no significant change was observed for the p-ERK1/2 expression (Fig. 4a). In addition, 2-AG alone could significantly activate p38 (3.84 $\pm 0.44, p<0.05$ ), but not ERK1/2 (Fig. 4a).

\section{2-AG suppressed activation of ERK $1 / 2$ and p38, and downregulation of GS induced by the late phase of LPS exposure}

Similarly, the effects of 2-AG on the activation of astrocytes induced by the late phase of LPS exposure were investigated. Astrocytes were pretreated with $0.01 \mu \mathrm{M} 2-\mathrm{AG}$ for $2 \mathrm{~h}$ and/or $1 \mu \mathrm{g} / \mathrm{ml}$ LPS for $12 \mathrm{~h}$ (Fig. 4b). Treatment of astrocytes with LPS for $12 \mathrm{~h}$ significantly increased the phosphorylation levels of ERK1/2 (5.45 \pm 0.22 for LPS vs. $1.00 \pm 0.21$ for control, $p<0.001)$ and p38 (25.53 \pm 1.85 for LPS vs. $1.00 \pm 0.31$ for control, $p<0.001$ ). Pretreatment with 2-AG significantly attenuated LPS-induced phosphorylation of ERK1/2 (2.87 \pm 0.19 for 2-AG plus LPS vs. $5.45 \pm 0.22$ for LPS, $p<0.001)$ and p38 (11.10 \pm 0.95 for 2-AG plus LPS vs. $25.53 \pm 1.85$ for LPS, $p<0.001)$. Stimulation of astrocytes by LPS for $12 \mathrm{~h}$ significantly decreased the expression level of GS $(0.42 \pm 0.04$ for LPS vs. $1.00 \pm 0.05$ for control, $p<0.001)$ and pretreatment with 2-AG reversed LPS-induced downregulation of GS expression $(0.85 \pm 0.06$ for 2 -AG plus LPS vs. $0.42 \pm 0.04$ for LPS, $p<0.001$ ).

\section{2-AG inhibited the translocations of MAPK and GS changes induced by LPS exposure}

Previous studies have demonstrated that p38 performs effects by translocating from cytoplasm to nucleus $[17,33]$. To investigate the effects of 2-AG on p38 translocation accompanying GS upregulation induced by the early phase of LPS exposure, astrocytes were pretreated with $0.01 \mu \mathrm{M}$ 2 -AG for $2 \mathrm{~h}$ and then exposed to $1 \mu \mathrm{g} / \mathrm{ml} \mathrm{LPS}$ for $2 \mathrm{~h}$, and p38 translocation and GS expression change were assessed using immunostaining assay (Fig. 5a). The results showed that pretreatment with 2-AG inhibited the nuclear translocation of p38 and cytoplasmic GS upregulation induced by early LPS exposure.

Similarly, to investigate the effects of 2-AG on ERK1/2 translocation accompanying GS downregulation induced by the late phase of LPS exposure, astrocytes were pretreated with $0.01 \mu \mathrm{M} 2$-AG for $2 \mathrm{~h}$ and then exposed to $1 \mu \mathrm{g} / \mathrm{ml}$ LPS for $12 \mathrm{~h}$, and the changes of ERK $1 / 2$ translocation and GS expression were assessed using immunostaining assay (Fig. 5b). The results showed that
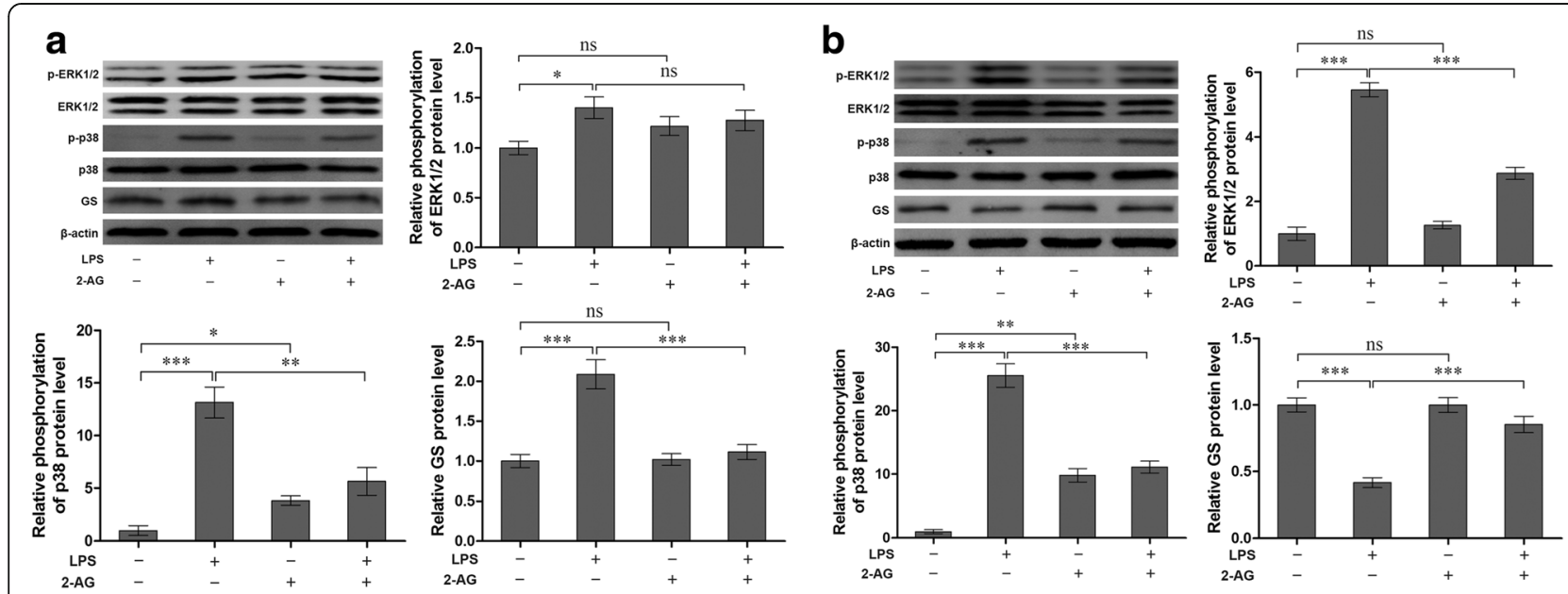

Fig. 4 Effects of 2-AG on phosphorylation of p38 (a), ERK1/2 (b), and GS expression (a, b) induced by LPS. Astrocytes were pretreated with $0.01 \mu \mathrm{M}$ 2-AG for $2 \mathrm{~h}$ and exposed to $1 \mu \mathrm{g} / \mathrm{ml}$ LPS for $2 \mathrm{~h}(\mathbf{a})$ or $12 \mathrm{~h}(\mathbf{b})$. The protein level of GS, p-p38 and p-ERK1/2 were measured, respectively $(\mathbf{a}, \mathbf{b})$. Error bars were \pm SD. $n=3 .{ }^{*} p<0.05,{ }^{* *} p<0.01$, and ${ }^{* * *} p<0.001$ 


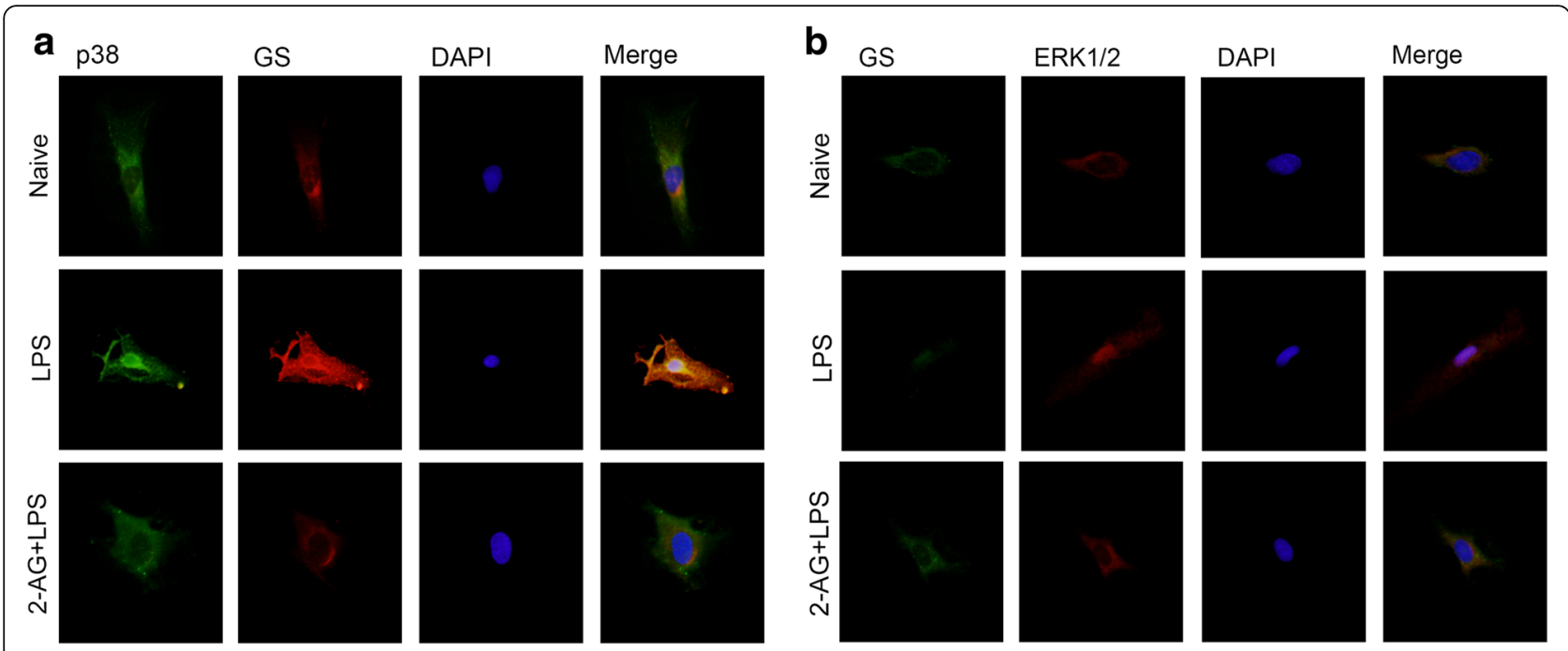

Fig. 5 Effects of 2-AG on translocation of p38 (a), ERK1/2 (b), and expression of GS (a, b) in astrocytes induced by LPS. Astrocytes were pretreated with $0.01 \mu \mathrm{M}$ 2-AG for $2 \mathrm{~h}$ and exposed to $1 \mu \mathrm{g} / \mathrm{ml}$ LPS for $2 \mathrm{~h}(\mathbf{a})$ or $12 \mathrm{~h}$ (b). Immunocytochemistry assay was used to analyze the translocation of p38 and ERK1/2 and the change of GS expression. Scar bar $=10 \mu \mathrm{m}$

pretreatment with 2-AG inhibited the nuclear translocation of ERK1/2 and cytoplasmic GS downregulation induced by late LPS exposure.

\section{2-AG inhibited upregulation of GS expression induced by} the early phase of LPS exposure via $\mathrm{CB}_{2} \mathrm{R}-\mathrm{p} 38$ pathway According to the above results, 2-AG could suppress the upregulation of GS induced by the early phase of LPS exposure via inhibiting the phosphorylation and translocation of $\mathrm{p} 38$. It has been demonstrated that two cannabinoid receptors, $\mathrm{CB}_{1} \mathrm{R}$ and $\mathrm{CB}_{2} \mathrm{R}$, were involved in the pharmacological effects of $2-\mathrm{AG}$ on glial cells [23]. To investigate which receptor mediates the modulation of GS by 2-AG, AM281 and AM630 were used to block the $C_{1} R$ and $C_{2} R$, respectively. The data showed that treatment of astrocytes with $1 \mu \mathrm{M}$ AM630 for $1 \mathrm{~h}$ significantly reversed the effects of 2-AG on the phosphorylation of p38 (2.46 \pm 0.07 for AM630 plus 2-AG and LPS vs. $1.55 \pm 0.13$ for 2-AG plus LPS, $p<0.01)$ and increase of GS $(2.02 \pm 0.08$ for AM630 plus 2-AG and LPS vs. $1.06 \pm 0.09$ for 2 -AG plus LPS, $p<0.01$ ) induced by the early phase of LPS exposure (Fig. 6a). $\mathrm{CB}_{1} \mathrm{R}$ antagonist, AM281, also could reverse the effect of 2-AG on GS upregulation $(1.94 \pm 0.09$ for AM281 plus 2-AG and LPS vs. $1.06 \pm$ 0.09 for 2-AG plus LPS, $p<0.01$ ), but not on phosphorylation of p38 induced by early LPS exposure $(1.29 \pm 0.12$ for AM630 plus 2-AG and LPS vs. $1.55 \pm$ 0.13 for 2-AG plus LPS, $p>0.05$ ) (Fig. 6a). These data suggested that $C_{2} R$ plays a significant role in the effect of 2-AG via p38-dependent signaling pathway, and activation of $\mathrm{CB}_{1} \mathrm{R}$ also could inhibit the increased GS expression via other uncertain signaling cascades.

\section{2-AG reversed downregulation of GS induced by late LPS exposure via $C B_{1} R / C B_{2} R-E R K 1 / 2$ pathways}

As shown in Fig. 6b, the effects of the late phase of LPS exposure on phosphorylation of ERK1/2 was reversed by 2-AG (1.27 \pm 0.07 for 2 -AG plus LPS vs. $2.14 \pm 0.15$ for LPS, $p<0.01$ ) while the effects of 2-AG was blocked by $\mathrm{CB}_{1} \mathrm{R}$ antagonist AM281 (2.18 \pm 0.09 for AM281 plus 2-AG and LPS vs. $1.27 \pm 0.07$ for 2 -AG plus LPS, $p$ $<0.01)$. Similarly, the effect of the late phase of LPS exposure on GS downregulation was reversed by 2-AG (1.01 \pm 0.05 for 2 -AG plus LPS vs. $0.44 \pm 0.04$ for LPS, $p$ $<0.001$ ) and the effect of 2-AG was blocked by $\mathrm{CB}_{1} \mathrm{R}$ antagonist AM281 $(0.63 \pm 0.06$ for AM281 plus 2-AG and LPS vs. $1.01 \pm 0.05$ for 2 -AG plus LPS, $p<0.01$ ). In addition, $\mathrm{CB}_{2} \mathrm{R}$ antagonist $\mathrm{AM} 630$ attenuated the effect of 2-AG on ERK1/2 phosphorylation (2.24 \pm 0.10 for AM630 plus 2-AG and LPS vs. $1.27 \pm 0.07$ for 2-AG plus LPS, $p<0.01)$ and GS downregulation $(0.47 \pm 0.08$ for AM630 plus 2-AG and LPS vs. $1.01 \pm 0.05$ for 2 -AG plus LPS, $p<0.01$; Fig. $6 \mathrm{~b}$ ) induced by the late phase of LPS exposure.

\section{Discussion}

GS plays a key role through preventing the excessive accumulation of ammonia and glutamate in synaptic surroundings, and thereby suppressing the development of glutamate/ammonia neurotoxicity [2, 34, 35]. Accumulating evidence demonstrated that alterations of GS expression in astrocytes are involved in a number of neurological disorders, including neuropathic pain $[3,5,7]$, 

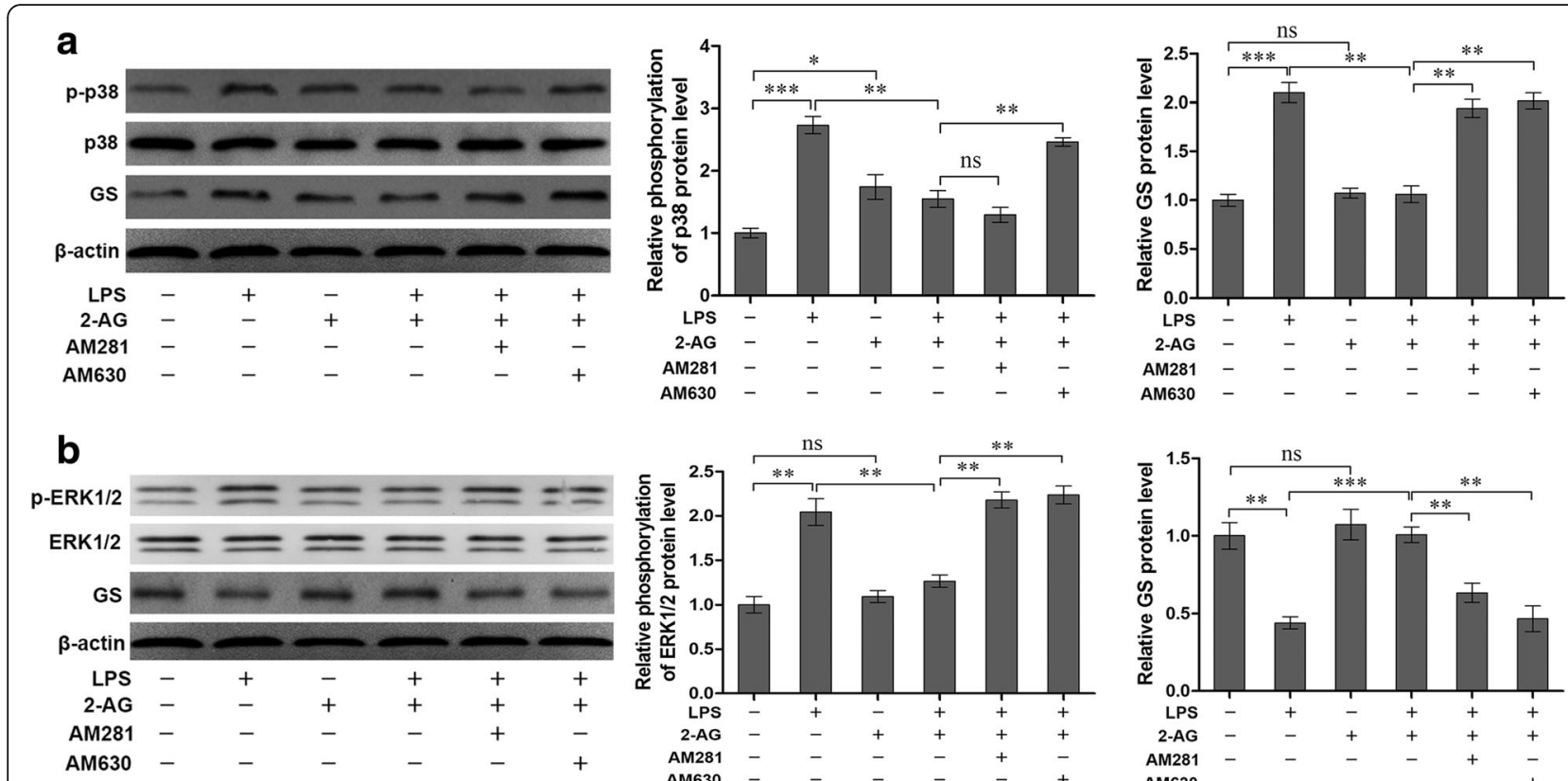

Fig. 6 Effects of inhibitors of $C B_{1} R$ and $\mathrm{CB}_{2} R$ antagonists on phosphorylation of p38 (a), ERK1/2 (b), and GS expression (a, b) in astrocytes induced by LPS. Astrocytes were pretreated with $1 \mu \mathrm{M}$ AM281 or AM630 for $1 \mathrm{~h}$ before cells were treated with 2-AG and LPS. The protein level of p-p38 (a), p-ERK1/2 (b), and GS were measured by western blotting. Error bars were \pm SD. $n=3 .{ }^{*} p<0.05,{ }^{* *} p<0.01$, and ${ }^{* *} p<0.001$

inflammatory pain [36], Alzheimer's disease, and Parkinson's disease $[6,8]$. Intriguingly, some diseases, such as hepatic encephalopathy, traumatic brain injury, and epilepsy, demonstrated both increase and decrease of GS while controlling GS expression can alleviate these diseases [4]. Consistently, the present study confirmed a biphasic change of GS expression in astrocytes after treatment with LPS. In addition, the present study found that LPS exposure induced increased of apoptosis and decrease of cell viability in the late stage of LPS exposure. These studies suggest that LPS induces a dynamic change of GS expression in astrocytes following the prolongation of LPS exposure time, which could modulate the survival of astrocytes. An improved understanding of the exact role of GS and mechanism in these neurological disorders and its involvement in pathology of diseases could enable the identification of innovative drugs to treat these diseases.

MAPK signaling, including p38 and ERK1/2, is a family of kinases involved in multiple physiological and pathological processes, including pain and neurodegenerative diseases [17-19]. In addition to neurons, MAPK signaling also exists in astrocytes and is activated under pathological stimulation [20-22]. The present study indicated that p38 and ERK1/2 were activated and translocated into nucleus in astrocytes by LPS exposure with different patterns, i.e., p38 was firstly activated at $30 \mathrm{~min}$ and reached peak at $2 \mathrm{~h}$ while ERK $1 / 2$ activated at $1 \mathrm{~h}$ and reached peak at $3 \mathrm{~h}$, while inhibition of p38 and ERK1/2 attenuated the changes of GS expression induced by LPS. These results suggested that there is a sequential activation of MPAK signaling in astrocytes, which plays an important role in modulating the GS expression. In addition, the present results were also consistent with previous study indicating that MAPK signaling is involved in the LPS-induced changes of GS in astrocytes [21].

2-AG is an endocannabinoid playing its roles in binding to $C_{1} R$ and $C_{2} R$ which are found to be expressed in astrocytes [23]. The present study indicated that 2-AG could modulate the GS expression and MAPK activation induced by LPS exposure through different CB receptors. Pascal et al. observed that 2-AG could activate p38 while blockade of $\mathrm{CB}_{1} \mathrm{R}$ could inhibit the effect of 2-AG on p38 [27], suggesting that 2-AG could modulate MAPK signaling in astrocytes. The results of present study indicated that, under the condition of the early phase of LPS exposure, activated p38 could translocate into nucleus, resulting in the increase of GS expression, and 2-AG could suppress the increase of GS by inhibiting the phosphorylation level and translocation of p38. While under the condition of late LPS exposure, activated ERK1/2 translocated into nucleus resulting in decrease of GS expression and 2-AG reversed the decrease of GS expression through reducing the activation and translocation of ERK1/2. It should be noted that although ERK1/2 and p38 were activated by early LPS exposure and late LPS exposure, respectively, the activation was relatively weaker when compared to late and 


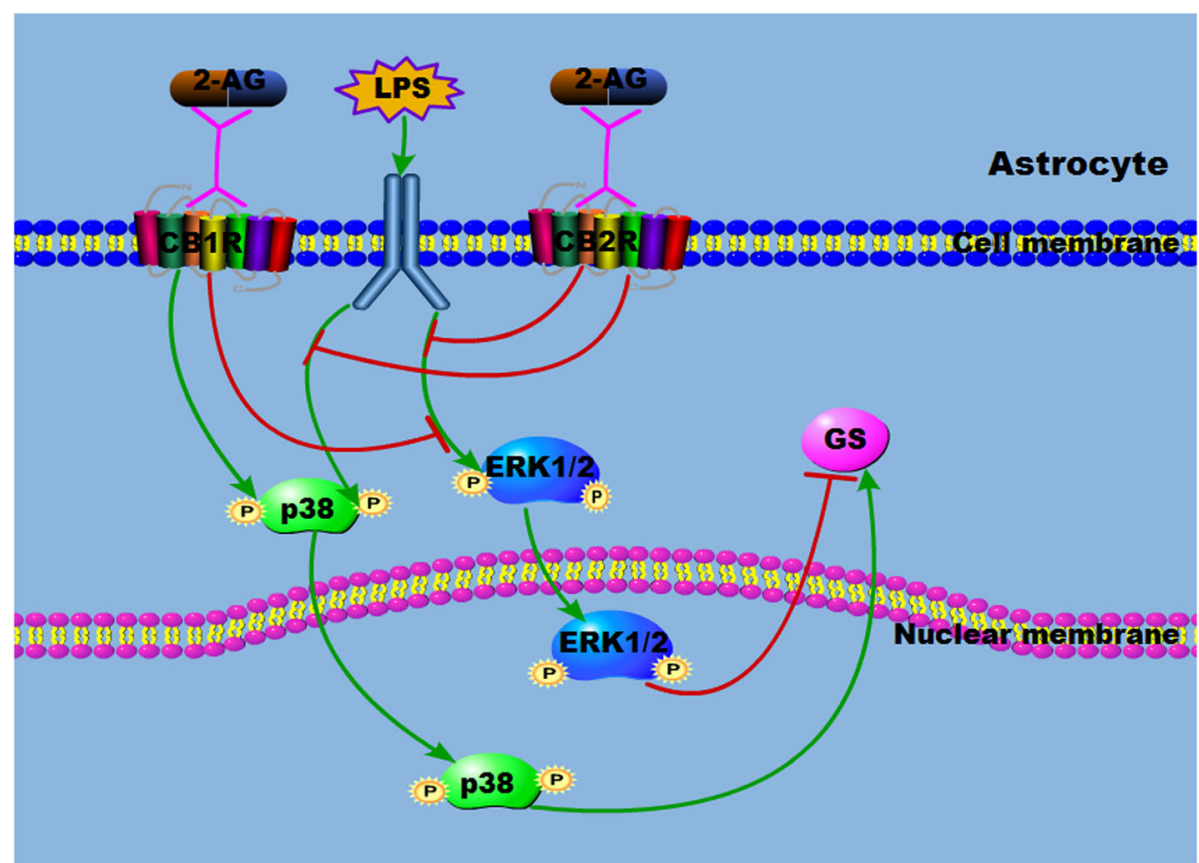

Fig. 7 Schematic drawing of the 2-AG on the GS expression and MAPK activation in astrocytes induced by LPS

early LPS exposure, respectively. These results indicated that 2-AG modulates the astrocyte survival by modulating p38 and ERK1/2 activation through different $C B$ receptors, which is supported by the results that 2-AG could prevent the apoptosis of astrocytes induced by LPS exposure.

\section{Conclusion}

The results of the present study indicated that LPS exposure for the short term and long term induced different changes of GS in astrocytes with activation of MAPK signaling, including p38 and ERK1/2 (Fig. 7). Endocannabinoid 2-AG modulates the GS expression induced by LPS exposure through $\mathrm{p} 38$ and ERK1/2 activation via different $\mathrm{CB}$ receptors to display the neuroprotection.

\section{Abbreviations}

2-AG: 2-arachidonyl glycerol; CBR: Cannabinoid receptor; ERK1/2: Extracellular signal-regulated protein kinase 1/2; GFAP: Glial fibrillary acidic protein; GS: Glutamine synthetase; LPS: Lipopolysaccharide; MAPK: Mitogen-activated protein kinase; MTT: Methyl thiazolyl tetrazolium

\section{Acknowledgements}

We thank Dr. Shengjun Fu, Dr. Jianzhong Lu, and Dr. Yan Tao from Key Laboratory of Urological Disease of Gansu Province for the equipment support.

\footnotetext{
Funding

This work was supported by the National Natural Science Foundation of China (Grant No. 81371230), Doctoral Research Foundation of Lanzhou University Second Hospital (Grant No. ynbskyjj2015-1-01), and Cuiying Scientific and Technological Innovation Program of Lanzhou University Second Hospital (Grant No. CY2017-QN12).
}

\section{Authors' contributions}

JW and BG conceived this experiment. SW and $\mathrm{HZ}$ conducted the experiment within XK's instruction. QX, WL, and YD analyzed the results. SW wrote the main manuscript. JW, BG, and YP revised the manuscript. All authors have reviewed the manuscript. All authors read and approved the final manuscript.

\section{Ethics approval}

All efforts were performed to minimize the number of neonatal rats used and their suffering. The procedures were approved by the Animal Care and the Ethic Committee of Animal Usage of Lanzhou University Second Hospital.

\section{Consent for publication}

Not applicable.

\section{Competing interests}

The authors declare that they have no competing interests.

\section{Publisher's Note}

Springer Nature remains neutral with regard to jurisdictional claims in published maps and institutional affiliations.

\section{Author details}

${ }^{1}$ Key Laboratory of Orthopaedics Disease of Gansu Province, Lanzhou University Second Hospital, No.82 Cuiyingmen Street, Lanzhou, Gansu 730030, People's Republic of China. ${ }^{2}$ Department of Orthopaedics, Lanzhou University Second Hospital, No.82 Cuiyingmen Street, Lanzhou, Gansu 730030, People's Republic of China. ${ }^{3}$ Clinical Laboratory, Lanzhou University Second Hospital, No.82 Cuiyingmen Street, Lanzhou, Gansu 730030, People's Republic of China.

Received: 22 February 2018 Accepted: 16 July 2018

Published online: 03 August 2018

\section{References}

1. Belanger M, Magistretti PJ. The role of astroglia in neuroprotection. Dialogues Clin Neurosci. 2009;11(3):281-95.

2. Cooper AJ. The role of glutamine synthetase and glutamate dehydrogenase in cerebral ammonia homeostasis. Neurochem Res. 2012;37(11):2439-55. 
3. Doyle T, et al. Targeting the overproduction of peroxynitrite for the prevention and reversal of paclitaxel-induced neuropathic pain. J Neurosci. 2012;32(18):6149-60.

4. Jayakumar AR, Norenberg MD. Glutamine synthetase: role in neurological disorders. Adv Neurobiol. 2016;13:327-50.

5. Mostafeezur RM, et al. Involvement of astroglial glutamate-glutamine shuttle in modulation of the jaw-opening reflex following infraorbital nerve injury. Eur J Neurosci. 2014;39(12):2050-9.

6. Olabarria $\mathrm{M}$, et al. Age-dependent decrease in glutamine synthetase expression in the hippocampal astroglia of the triple transgenic Alzheimer's disease mouse model: mechanism for deficient glutamatergic transmission? Mol Neurodegener. 2011;6:55.

7. Tsuboi Y, et al. Modulation of astroglial glutamine synthetase activity affects nociceptive behaviour and central sensitization of medullary dorsal horn nociceptive neurons in a rat model of chronic pulpitis. Eur J Neurosci. 2011;34(2):292-302.

8. Zipp F, et al. Glutamine synthetase activity in patients with Parkinson's disease. Acta Neurol Scand. 1998;97(5):300-2.

9. Marrs WR, et al. The serine hydrolase ABHD6 controls the accumulation and efficacy of 2-AG at cannabinoid receptors. Nat Neurosci. 2010;13(8):951-7.

10. Pertwee RG, et al. International Union of Basic and Clinical Pharmacology. LXXIX. Cannabinoid receptors and their ligands: beyond CB (1) and CB(2). Pharmacol Rev. 2010;62(4):588-631.

11. Chen X, Zhang J, Chen C. Endocannabinoid 2-arachidonoylglycerol protects neurons against beta-amyloid insults. Neuroscience. 2011;178:159-68.

12. Zhang J, Chen C. Endocannabinoid 2-arachidonoylglycerol protects neurons by limiting COX-2 elevation. J Biol Chem. 2008;283(33):22601-11.

13. Bisogno T, et al. Development of a potent inhibitor of $2-$ arachidonoylglycerol hydrolysis with antinociceptive activity in vivo. Biochim Biophys Acta. 2009;1791(1):53-60.

14. Costa B, et al. Oral anti-inflammatory activity of cannabidiol, a non-psychoactive constituent of cannabis, in acute carrageenan-induced inflammation in the rat paw. Naunyn Schmiedeberg's Arch Pharmacol. 2004;369(3):294-9.

15. Khasabova IA, et al. JZL184 is anti-hyperalgesic in a murine model of cisplatin-induced peripheral neuropathy. Pharmacol Res. 2014;90:67-75.

16. Pellkofer $\mathrm{HL}$, et al. The major brain endocannabinoid 2-AG controls neuropathic pain and mechanical hyperalgesia in patients with neuromyelitis optica. PLoS One. 2013;8(8):e71500.

17. Chang L, Karin M. Mammalian MAP kinase signalling cascades. Nature. 2001;410(6824):37-40

18. Kyriakis JM, Avruch J. Mammalian MAPK signal transduction pathways activated by stress and inflammation: a 10-year update. Physiol Rev. 2012;92(2):689-737.

19. Ji RR, et al. MAP kinase and pain. Brain Res Rev. 2009;60(1):135-48.

20. Gong P, et al. Phosphorylation of mitogen- and stress-activated protein kinase-1 in astrocytic inflammation: a possible role in inhibiting production of inflammatory cytokines. PLoS One. 2013;8(12):e81747.

21. Bellaver B, et al. Resveratrol protects hippocampal astrocytes against LPSinduced neurotoxicity through $\mathrm{HO}-1, \mathrm{p} 38$ and ERK pathways. Neurochem Res. 2015;40(8):1600-8

22. Huang W, et al. Paracrine factors secreted by MSCs promote astrocyte survival associated with GFAP downregulation after ischemic stroke via p38 MAPK and JNK. J Cell Physiol. 2015;230(10):2461-75.

23. Stella N. Cannabinoid and cannabinoid-like receptors in microglia, astrocytes, and astrocytomas. Glia. 2010;58(9):1017-30.

24. Molina-Holgado F, et al. Role of CB1 and CB2 receptors in the inhibitory effects of cannabinoids on lipopolysaccharide-induced nitric oxide release in astrocyte cultures. J Neurosci Res. 2002;67(6):829-36.

25. Guindon J, Hohmann AG. The endocannabinoid system and pain. CNS Neurol Disord Drug Targets. 2009;8(6):403-21.

26. Battista $\mathrm{N}$, et al. The endocannabinoid system in neurodegeneration. Ital J Biochem. 2006;55(3-4):283-9.

27. Derkinderen $P$, et al. Cannabinoids activate $\mathrm{p} 38$ mitogen-activated protein kinases through CB1 receptors in hippocampus. J Neurochem. 2001;77(3):957-60.

28. Bouaboula $M$, et al. Activation of mitogen-activated protein kinases by stimulation of the central cannabinoid receptor CB1. Biochem J. 1995;312(Pt 2):637-41.

29. Howlett AC. Cannabinoid receptor signaling. Handb Exp Pharmacol. 2005;168:53-79.

30. McCarthy KD, de Vellis J. Preparation of separate astroglial and oligodendroglial cell cultures from rat cerebral tissue. J Cell Biol. 1980;85(3):890-902.
31. Evren $V$, et al. Protective effect of edaravone against manganese-induced toxicity in cultured rat astrocytes. Environ Toxicol Pharmacol. 2015:40(2):563-7.

32. Zou J, et al. Down-regulation of glutamine synthetase enhances migration of rat astrocytes after in vitro injury. Neurochem Int. 2011;58(3):404-13.

33. Raingeaud J, et al. Pro-inflammatory cytokines and environmental stress cause p38 mitogen-activated protein kinase activation by dual phosphorylation on tyrosine and threonine. J Biol Chem. 1995;270(13):7420-6.

34. Wang XS, et al. Protective effects of Gastrodin against autophagy-mediated astrocyte death. Phytother Res. 2016;30(3):386-96.

35. Sharma A, Patro N, Patro IK. Lipopolysaccharide-induced apoptosis of astrocytes: therapeutic intervention by minocycline. Cell Mol Neurobiol. 2016;36(4):577-92.

36. Chiang CY, et al. Astroglial glutamate-glutamine shuttle is involved in central sensitization of nociceptive neurons in rat medullary dorsal horn. J Neurosci. 2007:27(34):9068-76.

\section{Ready to submit your research? Choose BMC and benefit from:}

- fast, convenient online submission

- thorough peer review by experienced researchers in your field

- rapid publication on acceptance

- support for research data, including large and complex data types

- gold Open Access which fosters wider collaboration and increased citations

- maximum visibility for your research: over $100 \mathrm{M}$ website views per year

At BMC, research is always in progress.

Learn more biomedcentral.com/submissions 